\title{
Characterization of cyclic Schur groups
}

\author{
Sergei Evdokimov \\ Steklov Institute of Mathematics \\ at St. Petersburg \\ evdokim@pdmi.ras.ru * \\ István Kovács \\ University of Primorska, Koper, Slovenia \\ kovacs@pef.upr.si ${ }^{\dagger}$ \\ Ilya Ponomarenko \\ Steklov Institute of Mathematics \\ at St. Petersburg \\ inp@pdmi.ras.ru $\ddagger$
}

\begin{abstract}
A finite group $G$ is called a Schur group, if any Schur ring over $G$ is associated in a natural way with a subgroup of $\operatorname{Sym}(G)$ that contains all right translations. It was proved by R. Pöschel (1974) that given a prime $p \geq 5$ a $p$-group is Schur if and only if it is cyclic. We prove that a cyclic group of order $n$ is Schur if and only if $n$ belongs to one of the following five families of integers: $p^{k}, p q^{k}, 2 p q^{k}, p q r, 2 p q r$ where $p, q, r$ are distinct primes, and $k \geq 0$ is an integer.
\end{abstract}

\footnotetext{
${ }^{*}$ The work was partially supported by Slovenian-Russian bilateral project, grant no. BI-RU/10-11-018.

${ }^{\dagger}$ The work was partially supported by Slovenian-Russian bilateral project, grant no. BI-RU/10-11-018.

†The work was partially supported by Slovenian-Russian bilateral project, grant no. BI-RU/10-11-018 and RFFI Grant 11-01-00760-a.
} 


\section{Introduction}

Let $G$ be a finite group. A subring of the group ring $\mathbb{Q} G$ is called a $S c h u r$ ring or $S$-ring over $G$, if it closed with respect to the componentwise multiplication and inversion. The first construction of such a ring was proposed by I. Schur 8 in connection with his famous result on permutation groups containing a regular cyclic subgroup. Namely, let $\Gamma$ be a permutation group on the set $G$ that contains the regular group $G_{\text {right }}$ induced by right multiplications,

$$
G_{\text {right }} \leq \Gamma \leq \operatorname{Sym}(G) .
$$

Denote by $\Gamma_{1}$ the stabilizer of the identity of $G$ in $\Gamma$. Then the submodule of $\mathbb{Q} G$ spanned by the $\Gamma_{1}$-orbits (transitivity module) is an S-ring over $G$. Such an S-ring was called schurian in [7. The general theory of S-rings was developed by $\mathrm{H}$. Wielandt in [9] where in particular he constructed an S-ring which cannot be obtained by the Schur method.

Definition (R. Pöschel). A finite group $G$ is called Schur, if any S-ring over $G$ is schurian.

The Wielandt example shows that not every finite group is Schur. More exactly, he proved that the group $\mathbb{Z}_{p} \times \mathbb{Z}_{p}$ is not Schur for prime $p \geq 5$. This fact was used by R. Pöschel in [7] to prove the following theorem.

Theorem. Any section of a Schur group is a Schur group. Moreover, for a prime $p \geq 5$ a $p$-group is Schur if and only if it is cyclic.

Since any finite nilpotent group is a direct product of its Sylow subgroups, we immediately obtain the following result.

Corollary. A nilpotent group of order coprime to 6 is Schur only if it is cyclic.

The above results show the importance of the cyclic case for the characterization of Schur groups. It should be noted that by the Pöschel theorem any cyclic $p$-group is Schur for $p \geq 5$. In fact, the schurity of cyclic 3 -groups was also proved in [7], whereas the same result for $p=2$ was obtained in [5]. However, till 2001 no cyclic non-Schur group was known, and moreover it was conjectured that all cyclic groups are Schur (the Schur-Klin conjecture). This conjecture had also been supported by the fact that the group $\mathbb{Z}_{n}$ where $n$ is a product of two distinct primes, is a Schur one [4. The first counterexamples to the conjecture were constructed in [1]; in all these examples $n$ was 
the product of at least four primes. Later in [3] the schurity of $\mathbb{Z}_{n}$ was proved when $n$ is the product of at most three primes or $n=p^{3} q$ where $p$ and $q$ are distinct primes. The main result of this paper completes the characterization of cyclic Schur groups.

Theorem 1.1 A cyclic group of order $n$ is Schur if and only if $n$ belongs to one of the following five (partially overlapped) families of integers:

$$
p^{k}, p q^{k}, 2 p q^{k}, p q r, 2 p q r
$$

where $p, q, r$ are distinct primes, and $k \geq 0$ is an integer.

Corollary 1.2 The minimum order of a cyclic non-Schur group equals 72 .

Let us briefly outline the proof of Theorem 1.1. To prove the necessity, for each integer $n$ satisfying the hypothesis of Theorem 2.1 we construct explicitly a non-schurian S-ring over a group $\mathbb{Z}_{n}$. This ring is the generalized wreath product of two smaller schurian S-rings each of which is in its turn the generalized wreath product of normal S-rings; the way is essentially the same as one used in [1]. It turns out (Lemma 2.2) that the complement to the set of all these $n$ coincides with the set of all numbers listed in (1).

To prove the sufficiency we have to verify that any S-ring over a cyclic group of order $n$ belonging to one of families (1), is schurian. We observe that any divisor of such $n$ also belongs to at least one of these families.

Definition 1.3 A non-schurian $S$-ring $\mathcal{A}$ over a group $G$ is called minimal if the $S$-ring $\mathcal{A}_{S}$ is schurian for any $\mathcal{A}$-section $S \neq G / 1$.

It is easily seen that any non-schurian S-ring contains a section the restriction to which is minimal non-schurian. Thus the sufficiency in Theorem 1.1 immediately follows from the theorem below.

Theorem 1.4 The order of the underlying group of a minimal non-schurian circulant S-ring cannot belong to any of families (1).

There are two key observations to prove Theorem 1.4 that are based on the results of [3]. The first is that any non-schurian circulant S-ring is a fusion of a quasidense 1 non-schurian circulant S-ring (Theorem 3.4). The second

\footnotetext{
${ }^{1}$ Quasidense circulant S-rings are introduced and studied in Section 3.
} 
is that such an S-ring is a proper generalized wreath product of two smaller schurian quasidense S-rings. Moreover, in the minimal case each of them is in its turn a proper generalized wreath product (Theorem 4.3). We use these observations in Sections 4 and 5 to exclude the first, second and fourth families, and the case $p=2$ in the other two families. The proof is completed in Section 6 by applying the criterion of schurity for S-rings of special form that was proved in Section 8. It should be mentioned that throughout the proof of Theorem 1.4 we use several auxiliary results on circulant S-rings that are collected in Section 7 .

In this paper we follow the notation and terminology of paper [3]. When referring to this paper we keep only the number of the statement, preceding it by the letter A (e.g. instead of [3, Theorem 4.1] we write Theorem A4.1). Several additional notations are listed below.

We write $\mathcal{A} \cong \mathcal{A}^{\prime}$ when S-rings $\mathcal{A}$ and $\mathcal{A}^{\prime}$ are Cayley isomorphic.

For an S-ring $\mathcal{A}$ and an $\mathcal{A}$-section $S$ we set $\operatorname{Hol}_{\mathcal{A}}(S)=\operatorname{Hol}(S) \cap \operatorname{Aut}\left(\mathcal{A}_{S}\right)$.

For an S-ring $\mathcal{A}$ over a group $G$ we set

$$
\mathcal{M}(\mathcal{A})=\left\{\Gamma \leq \operatorname{Aut}(\mathcal{A}): \Gamma \underset{2}{\approx} \operatorname{Aut}(\mathcal{A}) \text { and } G_{\text {right }} \leq \Gamma\right\}
$$

\section{Necessity in Theorem 1.1}

Here we prove the necessity of Theorem 1.1. Throughout this section $\mathbb{Z}_{n}$ is the additive group of integers modulo a positive integer $n$.

For any divisor $m$ of $n$ denote by $i_{m, n}: \mathbb{Z}_{m} \rightarrow \mathbb{Z}_{n}$ and $\pi_{n, m}: \mathbb{Z}_{n} \rightarrow \mathbb{Z}_{m}$ the group homomorphisms taking 1 to $n / m$ and to 1 respectively. Using them we identify the groups $i_{m, n}\left(\mathbb{Z}_{m}\right)$ and $\mathbb{Z}_{n} / \operatorname{ker}\left(\pi_{n, m}\right)$ with $\mathbb{Z}_{m}$. Thus every section of $\mathbb{Z}_{n}$ of order $m$ is identified with the group $\mathbb{Z}_{m}$. Moreover, the permutation $f \in \operatorname{Aut}\left(\mathbb{Z}_{n}\right)$ afforded by multiplication by an integer induces the permutation $f^{m} \in \operatorname{Aut}\left(\mathbb{Z}_{m}\right)$ afforded by multiplication by the same integer.

If $\mathcal{A}$ is an S-ring over $G=\mathbb{Z}_{n}$ and $H$ is the $\mathcal{A}$-group of order $m$, then $\mathcal{A}_{H}$ $\mathcal{A}_{G / H}$ are denoted respectively by $\mathcal{A}_{m}$ and $\mathcal{A}^{n / m}$. Let finally $\mathcal{A}_{i}$ be an S-ring over $\mathbb{Z}_{n_{i}}(i=1,2)$ and $\left(\mathcal{A}_{1}\right)^{m}=\left(\mathcal{A}_{2}\right)_{m}$ for some $m$ dividing both $n_{1}$ and $n_{2}$. Then the unique S-ring $\mathcal{A}$ over $\mathbb{Z}_{n_{1} n_{2} / m}$ from Theorem A3.4 is denoted by $\mathcal{A}_{1} 2_{m} \mathcal{A}_{2}$. We omit $m$ if $m=1$. 
Below given a positive integer $m$ we set

$$
\Omega^{*}(m)= \begin{cases}\Omega(m), & \text { if } m \text { is odd } \\ \Omega(m / 2), & \text { if } m \text { is even }\end{cases}
$$

where $\Omega(m)$ is the total number of prime factors of $m$. We observe that $\Omega(m) \leq 1$ if and only if $m$ is a divisor of twice a prime number.

Theorem 2.1 Let $n=n_{1} n_{2}$ where $n_{1}$ and $n_{2}$ are coprime positive integers such that $\Omega^{*}\left(n_{i}\right) \geq 2, i=1,2$. Then a cyclic group of order $n$ is not Schur.

Proof. Below for an integer $m \geq 3$ we denote by $K_{m}$ the subgroup of order 2 in the group $\operatorname{Aut}\left(\mathbb{Z}_{m}\right)$ that is generated by multiplication by -1 . Suppose first that $n_{1}=a b$ and $n_{2}=c d$ where $a, b, c, d \geq 3$ are integers. Set

$$
\begin{gathered}
\mathcal{A}_{1}=\operatorname{Cyc}\left(K_{a} \times K_{c}, \mathbb{Z}_{a c}\right), \quad \mathcal{A}_{2}=\operatorname{Cyc}\left(K_{b c}, \mathbb{Z}_{b c}\right), \\
\mathcal{A}_{3}=\operatorname{Cyc}\left(K_{a d}, \mathbb{Z}_{a d}\right), \quad \mathcal{A}_{4}=\operatorname{Cyc}\left(K_{b d}, \mathbb{Z}_{b d}\right) .
\end{gathered}
$$

It is easily seen that the group $\operatorname{Aut}\left(\mathcal{A}_{i}\right)$ is dihedral for $i=2,3,4$, and is the direct product of two dihedral groups for $i=1$. Therefore the S-ring $\mathcal{A}_{i}$ is normal for all $i$. Moreover, $\left(\mathcal{A}_{1}\right)^{c}=\operatorname{Cyc}\left(K_{c}, \mathbb{Z}_{c}\right)=\left(\mathcal{A}_{2}\right)_{c}$ and $\left(\mathcal{A}_{3}\right)^{d}=$ $\operatorname{Cyc}\left(K_{d}, \mathbb{Z}_{d}\right)=\left(\mathcal{A}_{4}\right)_{d}$. Thus one can form S-rings

$$
\mathcal{A}_{1,2}=\mathcal{A}_{1} \imath_{c} \mathcal{A}_{2} \quad \text { and } \quad \mathcal{A}_{3,4}=\mathcal{A}_{3} \imath_{d} \mathcal{A}_{4} .
$$

It is easily seen that $\left(\mathcal{A}_{1,2}\right)^{n_{1}}=\operatorname{Cyc}\left(K_{a}, \mathbb{Z}_{a}\right)$ \ $\operatorname{Cyc}\left(K_{b}, \mathbb{Z}_{b}\right)=\left(\mathcal{A}_{3,4}\right)_{n_{1}}$. Then

$$
\mathcal{A}:=\mathcal{A}_{1,2}{ }_{n_{1}} \mathcal{A}_{3,4}
$$

is an S-ring over $\mathbb{Z}_{n}$. Thus it suffices to verify that $\mathcal{A}$ is not schurian.

Suppose on the contrary that $\mathcal{A}$ is schurian. Then by Theorem A1.2 the S-rings $\mathcal{A}_{1,2}$ and $\mathcal{A}_{3,4}$ are schurian, and there exist groups $\Delta_{1,2} \in \mathcal{M}\left(\mathcal{A}_{1,2}\right)$ and $\Delta_{3,4} \in \mathcal{M}\left(\mathcal{A}_{3,4}\right)$ such that

$$
\left(\Delta_{1,2}\right)^{S}=\left(\Delta_{3,4}\right)^{S}
$$

where $S$ is the section of order $n_{1}$ used in the definition of the S-ring $\mathcal{A}$. In particular, for any permutation $f_{1} \in \Delta_{1,2}$ fixing 0 there exists a permutation $f_{2} \in \Delta_{3,4}$ fixing 0 and such that $f_{1}^{S}=f_{2}^{S}$. We claim: the permutation $\left(f_{1}\right)^{H}$ 
where $H$ is the group of order ac, is induced by multiplication by $\varepsilon \in\{1,-1\}$. However, if this is true, then the stabilizer of 0 in the group $\left(\Delta_{1,2}\right)^{H}$ is contained in $K_{a c}$. Therefore the basic set of the S-ring associated with the former group that contains 1 is of cardinality $\leq 2$. On the other hand, this $\mathrm{S}-$ ring coincides with $\mathcal{A}_{1}$ by the schurity of the S-ring $\mathcal{A}_{1,2}$ and the 2-equivalence of the groups $\Delta_{1,2}$ and $\operatorname{Aut}\left(\mathcal{A}_{1,2}\right)$. So the above basic set has cardinality 4 . Contradiction.

To prove the claim let $f_{1,1}$ and $f_{1,2}$ be the automorphisms of the S-rings $\mathcal{A}_{1}$ and $\mathcal{A}_{2}$ induced by $f_{1}$, and $f_{2,3}$ and $f_{2,4}$ the automorphisms of the S-rings $\mathcal{A}_{3}$ and $\mathcal{A}_{4}$ induced by $f_{2}$. Then the normality of these S-rings implies that $f_{1,1} \in K_{a} \times K_{c}, f_{1,2} \in K_{b c}$, and that $f_{2,3} \in K_{a d}$ and $f_{2,4} \in K_{b d}$. Clearly,

$$
\left(f_{1,1}\right)^{c}=\left(f_{1,2}\right)^{c} \quad \text { and } \quad\left(f_{2,3}\right)^{d}=\left(f_{2,4}\right)^{d}
$$

and due to the equality $\left(f_{1}\right)^{S}=\left(f_{2}\right)^{S}$ also

$$
\left(f_{1,1}\right)^{a}=\left(f_{2,3}\right)^{a} \quad \text { and } \quad\left(f_{1,2}\right)^{b}=\left(f_{2,4}\right)^{b} .
$$

Next, the permutations $f_{1,2}, f_{2,3}$ and $f_{2,4}$ are induced respectively by multiplications by some integers $\varepsilon_{1,2}, \varepsilon_{2,3}, \varepsilon_{2,4} \in\{1,-1\}$. Therefore, by the second equalities of (4) and (5) we have

$$
\varepsilon_{1,2}=\varepsilon_{2,3}=\varepsilon_{2,4}
$$

Denote this number by $\varepsilon$. Then by the first equalities of (44) and (5) the permutations $\left(f_{1,1}\right)^{a}$ and $\left(f_{1,1}\right)^{c}$, and hence the permutation $\left(f_{1}\right)^{H}$, are induced by multiplication by $\varepsilon$.

To complete the proof we observe that the theorem is proved in all cases except for the case when one of the numbers $n_{1}, n_{2}$, say $n_{1}$, is equal to 8 . Then obviously $n_{1}=a b / 2$ and $n_{2}=c d$ where $a=b=4$ and $c, d \geq 3$ are odd integers. Let us define S-rings $\mathcal{A}_{1}, \mathcal{A}_{2}, \mathcal{A}_{3}$ and $\mathcal{A}_{4}$ by formulas (2) and (3). Then again all these rings are normal,

$$
\left(\mathcal{A}_{1}\right)^{2 c}=\operatorname{Cyc}\left(K_{2 c}, \mathbb{Z}_{2 c}\right)=\left(\mathcal{A}_{2}\right)_{2 c}, \quad\left(\mathcal{A}_{3}\right)^{2 d}=\operatorname{Cyc}\left(K_{2 d}, \mathbb{Z}_{2 d}\right)=\left(\mathcal{A}_{4}\right)_{2 d},
$$

and one can form S-rings

$$
\mathcal{A}_{1,2}=\mathcal{A}_{1} 2_{2 c} \mathcal{A}_{2} \quad \text { and } \quad \mathcal{A}_{3,4}=\mathcal{A}_{3} 2_{2 d} \mathcal{A}_{4} \text {. }
$$


It should be stressed that $\mathcal{A}_{1,2}$ and $\mathcal{A}_{3,4}$ are S-rings over the groups $\mathbb{Z}_{c n_{1}}$ and $\mathbb{Z}_{d n_{1}}$. It is also easily seen that

$$
\left(\mathcal{A}_{1,2}\right)^{n_{1}}=\operatorname{Cyc}\left(K_{a}, \mathbb{Z}_{a}\right){ }_{2} \operatorname{Cyc}\left(K_{b}, \mathbb{Z}_{b}\right)=\left(\mathcal{A}_{3,4}\right)_{n_{1}} .
$$

Then

$$
\mathcal{A}:=\mathcal{A}_{1,2}{ }_{n_{1}} \mathcal{A}_{3,4}
$$

is an S-ring over $\mathbb{Z}_{n}$. Thus it suffices to verify that $\mathcal{A}$ is not schurian. The rest of the proof repeats the proof of the first part literally.

To complete the proof of the necessity we note that the required statement immediately follows from Theorem 2.1 and the lemma below.

Lemma 2.2 An integer $n$ belongs to none of the families listed in (11) if and only if $n=n_{1} n_{2}$ for some coprime positive integers $n_{1}$ and $n_{2}$ such that $\Omega^{*}\left(n_{i}\right) \geq 2, i=1,2$.

Proof. The sufficiency is straightforward by exaustive search. To prove the necessity let an integer $n=p_{1}^{k_{1}} \cdots p_{s}^{k_{s}}$ belong to none of families (1) where $p_{1}, \ldots, p_{s}$ are pairwise distinct primes. Then without loss of generality we can assume that

$$
2 \leq s \leq 4 \quad \text { and } \quad k_{1} \geq k_{2} \geq \cdots \geq k_{s}
$$

Suppose on the contrary that $n$ cannot be decomposed into the product of coprime positive integers $n_{1}$ and $n_{2}$ such that $\Omega^{*}\left(n_{i}\right) \geq 2, i=1,2$. Then $k_{2}=1$, for otherwise $s=2$ and $n$ belongs to the third family with $p=2$, which is impossible. Thus $k_{2}=\cdots=k_{s}=1$. Therefore $s=3$ or $s=4$, for otherwise $s=2$ and $n$ belongs to the second family. Let $s=3$. Then $k_{1} \neq 1$ because $n$ does not belong to the fourth family. So $k_{1} \geq 2$, and hence $2 \in\left\{p_{2}, p_{3}\right\}$ by the supposition. However, then $n$ belongs to the third family. Contradiction. Finally, let $s=4$. Then the supposition implies that $k_{1}=1$ and one of the $p_{i}$ 's equals 2 . But then $n$ belongs to the fifth family. Contradiction.

\section{$3 \quad$ Quasidense S-rings}

A circulant S-ring $\mathcal{A}$ is called quasidense, if any primitive $\mathcal{A}$-section is of prime order. Any dense S-ring is obviously quasidense. It is also clear that 
the property to be quasidense is preserved by taking the restriction to any $\mathcal{A}$-section. Moreover, in the quasidense case any minimal $\mathcal{A}$-group is of prime order, any maximal $\mathcal{A}$-group is of prime index, and the S-ring $\mathcal{A}_{S}$ is dense for any $\mathcal{A}$-section $S$ of prime power order.

Theorem 3.1 Any quasidense circulant S-ring with trivial radical is cyclotomic, and hence dense.

Proof. Let $\mathcal{A}$ be a quasidense circulant S-ring with trivial radical. Then $\mathcal{A}$ is the tensor product of a normal S-ring with trivial radical and S-rings of rank 2 by Theorem A4.1. However, any normal circulant S-ring is cyclotomic by Theorem A4.2. Besides, by the quasidensity the underlying group of any factor of rank 2 is of prime order. Therefore such a factor is also cyclotomic. Thus $\mathcal{A}$ is cyclotomic as the tensor product of cyclotomic S-rings.

The following two statements will be used in proving the sufficiency of Theorem 1.1 to find nontrivial $\mathcal{A}$-groups.

Corollary 3.2 Let $\mathcal{A}$ be a quasidense $S$-ring over a cyclic group $G$. Then any subgroup of $G$ that contains $\operatorname{rad}(\mathcal{A})$ is an $\mathcal{A}$-group. In particular, if $\operatorname{rad}(\mathcal{A})_{p}=1$ for a prime divisor $p$ of $|G|$, then $G_{p^{\prime}}$ is an $\mathcal{A}$-group.

Proof. The S-ring $\mathcal{A}_{G / L}$ where $L=\operatorname{rad}(\mathcal{A})$, has trivial radical. Therefore by Theorem 3.1 it is dense. Thus required statement follows from the fact that a group $H$ containing $L$ is an $\mathcal{A}$-group if and only if the group $H / L$ is an $\mathcal{A}_{G / L}$-group..

Corollary 3.3 Let $\mathcal{A}$ be a quasidense $S$-ring over a cyclic group $G$. Suppose that $\mathcal{A}$ is not the $U / L$-wreath product where $U / L$ is an $\mathcal{A}$-section such that the number $p:=|L|$ is prime. Then

(1) there exists $H \in \mathcal{G}(\mathcal{A})$ such that $H \not \leq U$ and $H_{p^{\prime}} \in \mathcal{G}(\mathcal{A})$,

(2) if $q:=|G / U|$ is a prime other than $p$, then $H_{p^{\prime}} \geq G_{q}$ for any group $H$ from statement (1).

Proof. To prove statement (1) we observe that by the hypothesis there exists $X \in \mathcal{S}(\mathcal{A})$ outside $U$ such that $\operatorname{rad}(X)_{p}=1$. Then $H=\langle X\rangle$ is an $\mathcal{A}$-group. Therefore the required statement follows from Corollary 3.2 applied to the Sring $\mathcal{A}_{H}$. Next, the condition of statement (2) implies that any group $H \not \leq U$ contains a generator of $G_{q}$. Therefore $H \geq G_{q}$ which proves this statement. 
The following theorem reduces the schurity problem for circulant S-rings to the quasidense case. The proof is based on the extension construction studied in [3].

Theorem 3.4 Given a circulant $S$-ring $\mathcal{A}$ there exists a quasidense $S$-ring $\mathcal{A}^{\prime} \geq \mathcal{A}$ such that $\mathcal{A}$ and $\mathcal{A}^{\prime}$ are schurian or not simultaneously.

Proof. Let us define an S-ring $\mathcal{A}^{\prime}$ recursively as follows. If $\mathcal{A}$ has no singular class of composite order, then we set $\mathcal{A}^{\prime}=\mathcal{A}$; otherwise we set

$$
\mathcal{A}^{\prime}=\left(\operatorname{Ext}_{C}(\mathcal{A}, \mathbb{Z} S)\right)^{\prime}
$$

where $C$ is a singular class of composite order and $S=S_{\min }(C)$. Then the S-ring $\mathcal{A}^{\prime}$ has no singular classes of composite order. Moreover, from Theorem A6.7 it follows that $\mathcal{A}$ and $\mathcal{A}^{\prime}$ are schurian or not simultaneously. To complete the proof let us verify that the S-ring $\mathcal{A}^{\prime}$ is quasidense. Suppose on the contrary that this is not true. Then there exists a primitive $\mathcal{A}^{\prime}$ section $S$ of composite order. Then by Theorem A4.6 the class of projectively equivalent $\mathcal{A}^{\prime}$-sections that contains $S$, is singular. Contradiction..

In general, the automorphism group of a quasidense S-ring is not solvable. However, from Theorem A8.1 it follows that in the schurian case such an Sring can always be obtained from an appropriate solvable permutation group in a standard way. The following theorem shows that "locally" this group has a rather simple form.

Theorem 3.5 Let $\mathcal{A}$ be a schurian quasidense circulant $S$-ring. Then there exists a group $\Gamma \in \mathcal{M}(\mathcal{A})$ such that $\Gamma^{S}=\operatorname{Hol}_{\mathcal{A}}(S)$ for any $\mathcal{A}$-section $S$ with $\operatorname{rad}\left(\mathcal{A}_{S}\right)=1$

Remark 3.6 In fact, we prove that the equality in the theorem statement holds for any $S$ such that $\mathcal{A}_{S}$ is the tensor product of a normal $S$-ring and S-rings of rank 2 .

Proof. The quasidensity of $\mathcal{A}$ implies that each primitive $\mathcal{A}$-section is of prime order. Therefore by Theorems A4.6 and A8.1 there exists a group $\Gamma \in \mathcal{M}(\mathcal{A})$ such that $\Gamma^{T} \leq \operatorname{Hol}(T)$ for all primitive $\mathcal{A}$-sections $T$. Let $S$ be an $\mathcal{A}$-section with $\operatorname{rad}\left(\mathcal{A}_{S}\right)=1$. Then by Theorem A4.1 the S-ring $\mathcal{A}_{S}$ is 
the tensor product of a normal S-ring, say $\mathcal{A}_{T_{0}}$, and S-rings of rank 2, say $\mathcal{A}_{T_{1}}, \ldots, \mathcal{A}_{T_{k}}$ where $T_{i}$ 's are $\mathcal{A}_{S^{-}}$groups. It follows that

$$
\Gamma^{T_{0}} \leq \operatorname{Aut}\left(\mathcal{A}_{T_{0}}\right) \leq \operatorname{Hol}\left(T_{0}\right)
$$

Moreover, by the above $\Gamma^{T_{i}} \leq \operatorname{Hol}\left(T_{i}\right)$ for all $i>0$, because the sections $T_{1}, \ldots, T_{k}$ are primitive. Thus

$$
\Gamma^{S} \leq \prod_{i=0}^{k} \Gamma^{T_{i}} \leq \prod_{i=0}^{k} \operatorname{Hol}\left(T_{i}\right)=\operatorname{Hol}(S) .
$$

But then $\Gamma^{S}$ is obviously a unique subgroup of $\operatorname{Hol}(S)$ in the set $\mathcal{M}\left(\mathcal{A}_{S}\right)$. Thus $\Gamma^{S}=\operatorname{Hol}_{\mathcal{A}}(S)$.

\section{Excluding families 1, 2 and 4}

In the end of this section we prove the following theorem showing that any minimal non-schurian quasidense $\mathrm{S}$-ring $\mathcal{A}$ over a cyclic group $G$ contains two distinct minimal $\mathcal{A}$-groups and two distinct maximal $\mathcal{A}$-groups, the relationship between which, is as in Fig. 1.

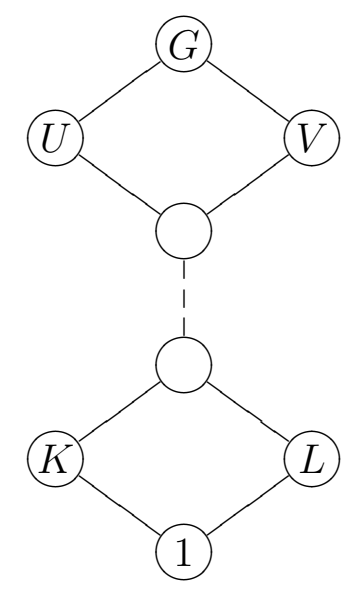

Figure 1:

Theorem 4.1 Let $\mathcal{A}$ be a minimal non-schurian quasidense S-ring over a cyclic group $G$ of order $n$ belonging to one of five families (1). Then 
(1) $n$ belongs to the third or fifth families,

(2) there exist distinct $\mathcal{A}$-groups $K, L$ of prime orders and distinct $\mathcal{A}$ groups $V$, $U$ of prime indices such that $L K \leq U \cap V$ and $\mathcal{A}$ is a proper $U / L$-wreath product.

We begin with studying minimal non-schurian circulant S-rings. In the following statement we establish general properties of them.

Lemma 4.2 Let $\mathcal{A}$ be a minimal non-schurian $S$-ring over a cyclic group $G$. Then

(1) $\mathcal{A}$ is a proper generalized wreath product,

(2) if $\mathcal{A}$ is a proper $U / L$-wreath product, then $\operatorname{rad}\left(\mathcal{A}_{U / L}\right) \neq 1$; moreover, $\left|\operatorname{rad}\left(\mathcal{A}_{U / L}\right)\right|>2$ whenever $\mathcal{A}_{U / L}$ is cyclotomic.

Proof. By Corollary A4.3 we can assume that $\operatorname{rad}(\mathcal{A}) \neq 1$. So statement $(1)$ follows from Theorem A4.1. The first part of statement (2) follows from the minimality of $\mathcal{A}$, Theorem A1.3 and Corollary A1.4. Similarly, to prove the second part of this statement it suffices to verify that the S-ring $\mathcal{A}_{U / L}$ is the tensor product of a normal S-ring and S-rings of rank 2 whenever it is cyclotomic and its radical is of order 2. However, under this condition the criterion of normality [2, Theorem 6.1] implies that $\mathcal{A}_{U / L}$ is not normal if and only if it is the tensor product one factor of which is an S-ring of rank 2 (over a cyclic group of prime order). Thus the required statement follows by induction.

By statement (1) of Lemma 4.2 any minimal non-schurian circulant S-ring is a proper generalized wreath product. The following important theorem shows that in the quasidense case both operands are also proper generalized wreath products.

Theorem 4.3 Let $\mathcal{A}$ be a minimal non-schurian quasidense S-ring over a cyclic group $G$ of order $n$ belonging to one of families (11). Then $\operatorname{rad}\left(\mathcal{A}_{U}\right) \neq 1$ and $\operatorname{rad}\left(\mathcal{A}_{G / L}\right) \neq 1$ whenever $\mathcal{A}$ is a proper $U / L$-wreath product.

Proof. Let $\mathcal{A}$ be a proper $U / L$-wreath product. Suppose on the contrary that $\operatorname{rad}\left(\mathcal{A}_{T}\right)=1$ where $T \in\{U, G / L\}$. Then the quasidensity of $\mathcal{A}$ implies by Theorem 3.1 that the S-ring $\mathcal{A}_{T}$, and hence the S-ring $\mathcal{A}_{S}$ with $S=U / L$, 
is cyclotomic. By the minimality of $\mathcal{A}$ and Lemma 4.2 this implies that $\left|\operatorname{rad}\left(A_{S}\right)\right|>2$. Thus $n$ does not belong to the fourth and the fifth families, because otherwise $|S|$ is either prime, or 4 , or the product of two distinct primes. Moreover, from Theorem 7.3 for $G=T$ it follows that $S_{l}=1$ for some odd prime divisor $l$ of $|T|$. Thus $n$ does not belong to the first family. In the remaining two cases the prime $l$ coincides with $p$, because otherwise $l=q$, and hence $|S|$ divides $2 p$ which is impossible by above. This proves the following lemma.

Lemma 4.4 Under the above assumptions we have $n=p q^{k}$ or $n=2 p q^{k}$, and $p \neq 2$. Moreover,

(1) if $\operatorname{rad}\left(\mathcal{A}_{U}\right)=1$, then $L_{p} \neq 1$,

(2) if $\operatorname{rad}\left(\mathcal{A}_{G / L}\right)=1$, then $(G / U)_{p} \neq 1$.

Let $\operatorname{rad}\left(\mathcal{A}_{U}\right)=1$. Assume that either $q=2$, or $G_{2^{\prime}}$ is not an $\mathcal{A}$-group. By Lemma 4.4 the number $|G / L|$ is a power of $q$ or twice a power of $q$. So by the assumption there is a unique maximal $\mathcal{A}_{G / L}$-group, say $U^{\prime} / L$. Therefore $U^{\prime} \geq U$, and hence $\mathcal{A}$ is the $U^{\prime} / L$-wreath product. Denote by $L^{\prime}$ a maximal possible $\mathcal{A}$-group containing $L$ for which $\mathcal{A}$ is the $U^{\prime} / L^{\prime}$-wreath product. Then the uniqueness of $U^{\prime}$ implies that $\operatorname{rad}\left(\mathcal{A}_{G / L^{\prime}}\right)=1$. (Indeed, otherwise by Theorem A4.1 the S-ring $\mathcal{A}$ is the $U^{\prime} / L^{\prime \prime}$-wreath product for some $L^{\prime \prime}>L^{\prime}$, which contradicts the maximality of $L^{\prime}$ ). Then by statement (2) of Lemma4.4 we conclude that $\left(G / U^{\prime}\right)_{p} \neq 1$. Taking into account that $L_{p}^{\prime} \geq L_{p} \neq 1$, we conclude that $p^{2}$ divides $n$ which is impossible by Lemma 4.4. This proves the first part of the following lemma (the second one is proved in a similar way).

Lemma 4.5 We have $n=2 p q^{k}$ and $q \neq 2$. Moreover,

(1) if $\operatorname{rad}\left(\mathcal{A}_{U}\right)=1$, then $G_{2^{\prime}}$ is an $\mathcal{A}$-group,

(2) if $\operatorname{rad}\left(\mathcal{A}_{G / L}\right)=1$, then $G_{2}$ is an $\mathcal{A}$-group.

To complete the proof of Theorem 4.3 we come to a contradiction under the assumption $T=U$ (the remaining case $T=G / L$ can be proved in a similar way). In this case we observe that $U^{\prime}:=G_{2^{\prime}}$ is an $\mathcal{A}$-group by Lemma 4.5. We claim that

$$
\mathcal{A}=\mathcal{A}_{U^{\prime} l_{U^{\prime} / L}} \mathcal{A}_{G / L}
$$


Indeed, since $\mathcal{A}$ is the $U / L$-wreath product, it suffices to verify that $U^{\prime} \geq U$. Suppose on the contrary that this is not true. Then the number $|U|$ must be even. This implies that $G_{2}$ is an $\mathcal{A}$-group (we used the fact that the S-ring $\mathcal{A}_{U}$ is cyclotomic, and hence dense). Since $p \neq 2$ and $q \neq 2$, this shows that $G_{2}$ is the $\mathcal{A}$-complement of $U^{\prime}$. Therefore by Corollary 7.2 we conclude that $\mathcal{A}=\mathcal{A}_{U^{\prime}} \otimes \mathcal{A}_{G_{2}}$. By the minimality of $\mathcal{A}$ this implies that the S-ring $\mathcal{A}$ is schurian. The obtained contradiction proves equality (6).

After increasing the group $L$ in (6) (if necessary), we can assume that it is a maximal possible $\mathcal{A}$-group with that property. Then

$$
(G / L)_{p}=1 \quad \text { and } \quad(G / L)_{2} \notin \mathcal{G}\left(\mathcal{A}_{G / L}\right) .
$$

The first equality follows from Lemma 4.4. To prove the second one suppose on the contrary that the group $(G / L)_{2}$ is the $\mathcal{A}_{G / L^{-}}$complement of $U^{\prime} / L$. Therefore by Corollary 7.2 we conclude that $\mathcal{A}_{G / L}=\mathcal{A}_{U^{\prime} / L} \otimes \mathcal{A}_{(G / L)_{2}}$. By the minimality of $\mathcal{A}$ and Theorem 7.5 this implies that the S-ring $\mathcal{A}$ is schurian. The obtained contradiction proves (7).

Due to the quasidensity of $\mathcal{A}$ formula (7) implies that there is the only

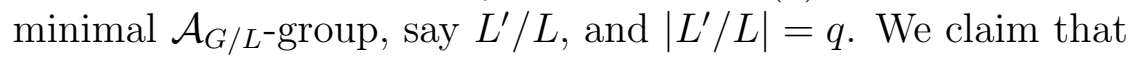

$$
\mathcal{A}_{G / L}=\mathcal{A}_{U^{\prime} / L} \ell_{U^{\prime} / L^{\prime}} \mathcal{A}_{G / L^{\prime}} .
$$

Indeed, otherwise by Corollary 3.3 there exists an $\mathcal{A}_{G / L}$-group $H / L$ such that

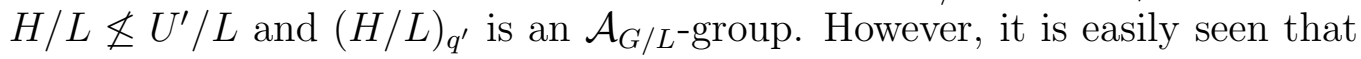
in our case $(H / L)_{q^{\prime}}=(G / L)_{2}$, which contradicts the second relation in (7). The obtained contradiction proves (8) .

Equalities (6) and (8) show that the S-ring $\mathcal{A}$ is the $U^{\prime} / L^{\prime}$-wreath product. However, this is impossible by the maximality of $L$.

Proof of Theorem 4.1. Statement (1) immediately follows from statement (2). To prove the latter we observe that by Lemma 4.2 the S-ring $\mathcal{A}$ is a proper $U / L$-wreath product for some $\mathcal{A}$-groups $U$ and $L$. By the quasidensity of $\mathcal{A}$ we can assume that $L$ is of prime order and $U$ is of prime index. Denote by $\widetilde{U}$ a minimal subgoup of $U$ such that the S-ring $\mathcal{A}$ is the $\widetilde{U} / L$-wreath product. Then by Theorem 4.3 the S-ring $\mathcal{A}_{\widetilde{U}}$ is a proper $U^{\prime} / K$ wreath product for some $\mathcal{A}$-groups $U^{\prime}$ and $K$. Again we can assume that $K$ is of prime order. By the minimality of $\widetilde{U}$ we conclude that $K \neq L$. Besides, $K L \leq U$ because $L \leq U$ and $K \leq U^{\prime}<\widetilde{U} \leq U$. 
Similarly, denote by $\widetilde{L}$ a maximal subgroup of $U$ that contains $L$ and such that the S-ring $\mathcal{A}$ is the $U / \widetilde{L}$-wreath product. Then again by Theorem 4.3 the S-ring $\mathcal{A}_{G / \widetilde{L}}$ is a proper $V / L^{\prime}$-wreath product for some $\mathcal{A}$-groups $V$ and $L^{\prime}$ such that $V$ is of prime index in $G$. By the maximality of $\widetilde{L}$ we conclude that $V \neq U$. Besides, obviously $V \geq L$. To complete the proof it suffices to note that $K \leq V$. Indeed, if this is not true, then any nontrivial basic set of $\mathcal{A}_{G / \widetilde{L}}$ inside $K \widetilde{L} / \widetilde{L}$, is outside $V / \widetilde{L}$ and has trivial radical (because $|K \widetilde{L} / \widetilde{L}|=|K|$ is prime), which is impossible.

\section{Excluding families 3 and 5 for $\mathrm{p} \neq \mathbf{2}$}

In the end of this section we prove the following theorem that will enable us to exclude the cases in the title.

Theorem 5.1 Let $\mathcal{A}$ be a minimal non-schurian quasidense S-ring over a cyclic group $G$ of order $n$ belonging to the third or fifth of families (1). Then

(1) $p=2$,

(2) $\mathcal{A}$ is both $U / L$ - and $V / K$-wreath product where $K, L, U, V$ are $\mathcal{A}$ groups defined by

(1) $|K|=2,|L|=q,|U|=2 q r,|V|=4 q$ for $n=4 q r$,

(2) $|K|=2,|L|=q,|U|=2 q^{k},|V|=4 q^{k-1}$ for $n=4 q^{k}$,

with $q$ and $r$ distinct odd primes and $k \geq 2$.

Throughout the rest of the section $\mathcal{A}$ denotes an S-ring satisfying the hypothesis of Theorem 5.1. It is also assumed that we are given $\mathcal{A}$-groups $K, L, U, V$ for which statement (2) of Theorem 4.1 holds.

Theorem 5.2 The number $|U / L|$ is even.

Proof. Suppose on the contrary that $|U / L|$ is odd. Then either $|L|=2$, or $|G / U|=2$. Let us consider the former case, the latter one can be proved similarly. We are to find an $\mathcal{A}$-group $U^{\prime}$ such that $L \leq U^{\prime} \leq U$ and

$$
\mathcal{A}=\mathcal{A}_{U^{\prime}{ }_{U^{\prime} / L}} \mathcal{A}_{G / L} \quad \text { and } \quad\left(U^{\prime}\right)_{2^{\prime}} \in \mathcal{G}(\mathcal{A}) .
$$


Indeed, if such a group does exist, then by Corollary 7.2 with $G=U^{\prime}$, $H=\left(U^{\prime}\right)_{2^{\prime}}, S=L / 1$ and $T=U^{\prime} / L$, we obtain that $\mathcal{A}_{U}=\mathcal{A}_{L} \otimes \mathcal{A}_{U^{\prime} / L}$. By the minimality of $\mathcal{A}$ and Theorem 7.5 with $U=U^{\prime}$ this implies that the S-ring $\mathcal{A}$ is schurian which is not true.

In the case $n=2 p q r$ set $U^{\prime}=U$. Then the left-hand side relation in (9) is obvious. To prove the other one we observe that by Theorem A11.4 the number $|U|$ is the product of three primes, the S-ring $\mathcal{A}_{U}$ is not a proper wreath product and $\mathcal{A}_{U / L}$ is a proper wreath product. So, since the number $|U / L|$ is odd, the hypothesis of Lemma A11.2 is satisfied for $\mathcal{A}=\mathcal{A}_{U}, S=$ $U / L$ and $r=2$. By this lemma we obtain that $U_{2^{\prime}} \in \mathcal{G}\left(\mathcal{A}_{U}\right)$, and we are done.

In the case $n=2 p q^{k}$ set $U^{\prime}$ to be a minimal $\mathcal{A}$-subgroup of $U$, for which the first relation in (9) holds. We can assume that

$$
\left(U^{\prime}\right)_{p} \neq 1
$$

Indeed, otherwise $\left|U^{\prime}\right|=2 q^{i}$ for some $i$. Moreover, the minimality of $U^{\prime}$ implies that the S-ring $\mathcal{A}_{U^{\prime}}$ is not the $U^{\prime \prime} / L$-wreath product where $U^{\prime \prime}$ is the subgroup of $U^{\prime}$ of index $q$. Then by Corollary 3.3 with $p=2$ there exists

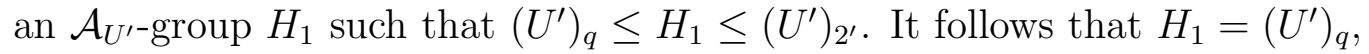
and the second relation in (9) holds.

From (10) it follows that $|G / V|=p$, and taking into account that $|U / L|$ is odd, also that $p$ is odd. Therefore $U^{\prime} \cap V \neq U^{\prime}$. By the minimality of $U^{\prime}$ this implies that the S-ring $\mathcal{A}_{U^{\prime}}$ is not a $\left(U^{\prime} \cap V\right) / L$-wreath product. So by Corollary 3.3 with $(p, q)=(2, p)$ there exists an $\mathcal{A}$-group $H_{1}$ such that

$$
\left(U^{\prime}\right)_{p} \leq H_{1} \leq\left(U^{\prime}\right)_{2^{\prime}}
$$

Denote by $H$ a maximal $\mathcal{A}$-subgroup of $U^{\prime}$ that contains $H_{1}$. Then due to (11) we have $\left|U^{\prime} / H\right| \in\{2, q\}$. If $\left|U^{\prime} / H\right|=2$, then the second relation in (9) holds and we are done. Finally, if $\left|U^{\prime} / H\right|=q$ then due to the minimality of $U^{\prime}$ the S-ring $\mathcal{A}_{U^{\prime}}$, is not an $H / L$-wreath product. By Corollary 3.3 with $(p, q)=(2, q)$ there exists an $\mathcal{A}$-group $H$ such that

$$
\left(U^{\prime}\right)_{q} \leq H_{2} \leq\left(U^{\prime}\right)_{2^{\prime}}
$$

Thus by (11) and (12) we have $\left(U^{\prime}\right)_{2^{\prime}}=H_{1} H_{2}$, and hence $\left(U^{\prime}\right)_{2^{\prime}} \in \mathcal{G}(\mathcal{A})$.

Theorem 5.3 The order of $G$ is divisible by 4 . 
Proof. Suppose first that $n=2 p q r$. Then by statement (1) of Theorem A11.4 (where the lattice of $\mathcal{A}$-groups is found) there are exactly two maximal $\mathcal{A}$ groups and exactly two minimal $\mathcal{A}$-groups; the former are of prime index whereas the latter are of prime order. From statement (2) of Theorem 4.1 it follows that these groups are $U, V$ and $K, L$ respectively, and also that

$$
|U / L| \cdot|V / K|=|G|
$$

On the other hand, we claim that the S-rings $\mathcal{A}_{U}$ and $\mathcal{A}_{G / L}$ are non-normal. Indeed, otherwise by statement (3) of Theorem A11.4 the number $|U / L|$ is a square of a prime. In our case this is possible only for $p=2$. But in this case $|U / L|=4$ which is impossible by the latter theorem. The claim is proved. So by statement (5) of the same theorem the S-ring $\mathcal{A}$ is the $V / K$-wreath product. Then by Theorem 5.2 of this paper the number $|V / K|$ is even. Thus 4 divides $|G|$.

Let $n=2 p q^{k}$. Suppose on the contrary that $p \neq 2$. Then since $q$ is odd and $|U / L|$ is even (Theorem [5.2), from statement (2) of Theorem 4.1 it follows that $|G / V|=2$ or $|K|=2$. Let us consider the former case, the latter one can be proved similarly. In this case $|V|$ is odd. Therefore by Theorem 5.2 the S-ring $\mathcal{A}$ is neither $V / K$ - nor $V / L$-wreath product. So by Corollary 3.3 with $(p, q)=(2, p)$ and $(p, q)=(2, q)$ there exist $\mathcal{A}$-groups $H_{1}$ and $H_{2}$ such that

$$
G_{2} \leq H_{1} \leq G_{p^{\prime}} \text { and } \quad G_{2} \leq H_{2} \leq G_{q^{\prime}}
$$

Thus $G_{2}=H_{1} \cap H_{2}$, and hence $G_{2}$ is an $\mathcal{A}$-group. By Corollary 7.2 this implies that $\mathcal{A}=\mathcal{A}_{G_{2}} \otimes \mathcal{A}_{V}$ which is impossible by the minimality of $\mathcal{A}$..

Theorem 5.4 Without loss of generality we can assume that 4 does not divide $|U / L|$.

Proof. By Theorem 5.3 we have $p=2$. So $n=4 q r$ or $n=4 q^{k}$. In the former case $|U / L|$ is divisible by 4 only if $|U / L|=4$. However, in this case the S-ring $\mathcal{A}_{U / L}$ is cyclotomic and $\left|\operatorname{rad}\left(\mathcal{A}_{U / L}\right)\right| \leq 2$, which contradicts statement (2) of Lemma 4.2. Thus we can assume that $n=4 q^{k}$ and 4 divides $|U / L|$. Then from statement (2) of Theorem 4.1 it follows that

$$
|G / U|=|L|=q \quad \text { and } \quad|G / V|=|K|=2 .
$$


Therefore it suffices to verify that the S-ring $\mathcal{A}$ is either $U / K$ - or $V / L$-wreath product. Suppose on the contrary that this is not true. Then by Corollary 3.3 with $(p, q)=(2, q)$ and $(p, q)=(q, 2)$ there exist $\mathcal{A}$-groups $H_{1}$ and $H_{2}$ such that

$$
G_{q} \leq H_{1} \leq G_{2^{\prime}} \text { and } \quad G_{2} \leq H_{2} \leq G_{q^{\prime}}
$$

It follows that $H_{1}=G_{q}$ and $H_{2}=G_{2}$. Thus $G_{q}$ and $G_{2}$ are $\mathcal{A}$-groups. By the quasidensity of $\mathcal{A}$ this implies that $\mathcal{A}$ is dense.

Denote by $\widetilde{U}$ the minimal $\mathcal{A}$-subgroup of $U$, for which the S-ring $\mathcal{A}$ is the $\widetilde{U} / L$-wreath product. Then by Theorem 4.3 with $U=\widetilde{U}$ the radical of the ring $\mathcal{A}_{\widetilde{U}}$ is nontrivial. Since this S-ring is dense, from [6, Theorem 3.4] (see also statement (1) of [2, Theorem 5.4]) it follows that it is a $U^{\prime} / L^{\prime}$-wreath product where the number $\left|L^{\prime}\right|=\left|\widetilde{U} / U^{\prime}\right|$ is the greatest prime divisor of $\left|\operatorname{rad}\left(\mathcal{A}_{\widetilde{U}}\right)\right|$. By the minimality of the group $\widetilde{U}$ we conclude that this prime divisor is equal to 2 . Thus

$$
L^{\prime}=K=\operatorname{rad}\left(\mathcal{A}_{\widetilde{U}}\right) \quad \text { and } \quad\left|\widetilde{U} / U^{\prime}\right|=2
$$

By Corollary 7.2 we have $\mathcal{A}_{U^{\prime}}=\mathcal{A}_{K} \otimes \mathcal{A}_{U_{q}^{\prime}}$ and $\mathcal{A}_{\widetilde{U} / K}=\mathcal{A}_{\widetilde{U}_{2} / K} \otimes \mathcal{A}_{U^{\prime} / K}$. Since $\mathcal{A}_{\widetilde{U}}$ is the $U^{\prime} / K$-wreath product this implies that

$$
\mathcal{A}_{\widetilde{U}}=\mathcal{A}_{\widetilde{U}_{2}} \otimes \mathcal{A}_{\widetilde{U}_{q}}
$$

Therefore $\mathcal{A}_{\widetilde{U} / L} \cong \mathcal{A}_{\widetilde{U}_{2}} \otimes \mathcal{A}_{\widetilde{U}_{q} / L}$. Moreover, the S-ring $\mathcal{A}_{\widetilde{U}_{2}}$ being a dense S-ring over a cyclic group of order 4 , is cyclotomic and $\left|\operatorname{rad}\left(\mathcal{A}_{\widetilde{U}_{2}}\right)\right| \leq 2$. Finally, by Corollary 7.4 the S-ring $\mathcal{A}_{\widetilde{U}_{q} / L}$ has trivial radical because by (14) and (15) so is the S-ring $\mathcal{A}_{\widetilde{U}_{q}}$. Therefore the latter S-ring is cyclotomic by Theorem 3.1. Thus the S-ring $\mathcal{A}_{\widetilde{U} / L}$ is cyclotomic and $\left|\operatorname{rad}\left(\mathcal{A}_{\widetilde{U} / L}\right)\right| \leq 2$ which contradicts statement (2) of Lemma 4.2.

Proof of Theorem 5.1. By Theorem 4.1 we can assume that the hypothesis under which Theorems [5.2, 5.3 and 5.4 were proved, holds for the S-ring $\mathcal{A}$. Then statement (1) immediately follows from Theorem 5.3. Thus

$$
n=4 q r \quad \text { or } \quad n=4 q^{k}
$$

where $q$ and $r$ distinct odd primes and $k \geq 2$. To prove statement (2) choose the groups $K, L, U, V$ as above. Then obviously $|K L|=2 q$ when $n=4 q^{k}$. The same is also true for $n=4 q r$ after interchanging $q$ and $r$ (if necessary). By Theorems 5.2 and 5.4 we can also assume $|U / L|=2(\bmod 4)$. 
Let $n=4 q r$. Then by the above assumptions the number $|U / L|$ is not a prime square. By statement (3) of Theorem A11.4 this implies that neither of the S-rings $\mathcal{A}_{U}$ and $\mathcal{A}_{G / L}$ is normal. Therefore by statement (5) of that theorem the S-ring $\mathcal{A}$ is the $V / K$-wreath product. Besides $|V / K|=2$ ( mod 4). Thus without loss of generality we can assume that $|K|=2$. Then $|L|=q$ and $|G / U|=2$. It follows that $|U|=2 q r$ and $|V|=4 q$, which completes the proof in this case.

Let $n=4 q^{k}$. Then by the above assumptions one of the following holds:

(1) $|K|=2,|L|=q,|U|=2 q^{k},|V|=4 q^{k-1}$,

(2) $|K|=q,|L|=2,|U|=4 q^{k-1},|V|=2 q^{k}$.

Thus it suffices to verify that $\mathcal{A}$ is the $V / K$-wreath product. Suppose that this is not true.

In case (1) by Corollary 3.3 with $p=2$ there exists an $\mathcal{A}$-group $H_{1}$ such that $G_{q} \leq H_{1} \leq G_{2^{\prime}}$. It follows that $H_{1}=G_{q}$, and hence $G_{q}$ is an $\mathcal{A}$-group. By Corollary 7.2 this implies that

$$
\mathcal{A}_{U}=\mathcal{A}_{G_{q}} \otimes \mathcal{A}_{K} .
$$

Denote by $H$ the maximal $\mathcal{A}$-group such that $L \leq H \leq G_{q}$ and $\operatorname{rad}\left(\mathcal{A}_{H}\right)=1$. Set $U^{\prime}=H K$. Then the radical of any basic set inside $U \backslash U^{\prime}$ contains $L$. Since the same is true also for any basic set outside $U$, the S-ring $\mathcal{A}$ is the $U^{\prime} / L$-wreath product. Besides, $\operatorname{rad}\left(\mathcal{A}_{U^{\prime}}\right)=1$ because $\mathcal{A}_{U^{\prime}}=\mathcal{A}_{H} \otimes \mathcal{A}_{K}$ and $|K|=2$. By statement (2) of Theorem 7.3 with $G=U^{\prime}$ this implies that $\operatorname{rad}\left(\mathcal{A}_{U^{\prime} / L}\right)=1$. However, this contradicts statement (2) of Lemma 4.2. Thus case (1) is impossible.

In case (2) one can similarly prove that $G_{2}$ is an $\mathcal{A}$-group, and $\mathcal{A}_{G / L}=$ $\mathcal{A}_{G_{2} / L} \otimes \mathcal{A}_{V / L}$. It is also easily seen that any basic set outside $U$ is highest in $\mathcal{A}$ or in $\mathcal{A}_{V}$. Therefore the S-ring $\mathcal{A}$ is the $U / L^{\prime}$-wreath product where $L^{\prime}=\operatorname{rad}\left(\mathcal{A}_{V}\right)$. Besides, $\operatorname{rad}\left(\mathcal{A}_{G / L^{\prime}}\right)=1$ because $\mathcal{A}_{G / L^{\prime}}=\mathcal{A}_{H / L^{\prime}} \otimes \mathcal{A}_{V / L^{\prime}}$ and $\left|H / L^{\prime}\right|=2$, where $H=G_{2} L^{\prime}$. By statement (2) of Theorem 7.3 with $G=G / L^{\prime}$ this implies that $\operatorname{rad}\left(\mathcal{A}_{U / L^{\prime}}\right)=1$. However, this is impossible by statement (2) of Lemma 4.2. 


\section{Proof of Theorem 1.4}

Let $\mathcal{A}$ be a minimal non-schurian S-ring over a cyclic group $G$ of order $n$. Suppose on the contrary that $n$ belongs to one of families (11). Since any divisor of $n$ also belongs to one of these families, by statement (3) of Lemma 4.2 without loss of generality we can assume that $\mathcal{A}$ is quasidense. Then by Theorems 4.1 and 5.1 we have $n=4 q r$ or $n=4 q^{k}$, and $\mathcal{A}$ is both $U / L$ - and $V / K$-wreath product where $K, L, U, V$ are $\mathcal{A}$-groups defined by

(1) $|K|=2,|L|=q,|U|=2 q r,|V|=4 q$ for $n=4 q r$,

(2) $|K|=2,|L|=q,|U|=2 q^{k},|V|=4 q^{k-1}$ for $n=4 q^{k}$,

with $q$ and $r$ distinct odd primes and $k \geq 2$. In both cases we will verify that the hypothesis of Theorem 8.1 is satisfied for some $\mathcal{A}$-groups so that the generalized wreath product for $\mathcal{A}$ defined there is proper. Then by that theorem $\mathcal{A}$ is schurian because due to the minimality of $\mathcal{A}$ so are the operands of this product. Contradiction.

Suppose that we are in case (1). Set $H_{1}=H_{2}=H$ where $H=K L=$ $U \cap V$. First, we observe that relations (23) and (24) are obviously satisfied. Furthermore, $|U / L|=2 r$ is not a prime square, and hence by statement (3) of Theorem A11.4 the S-rings $\mathcal{A}_{U}$ and $\mathcal{A}_{G / L}$ are not normal. By statement (4) of that theorem this implies that these S-rings are respectively the $H / K$ and $V / H$-wreath products. Besides, condition (1) of Theorem 8.1 is trivially satisfied because the underlying groups of the S-rings $\mathcal{A}_{H / K}, \mathcal{A}_{U / H}, \mathcal{A}_{V / H}$ and $\mathcal{A}_{H / L}$, are of prime orders, whereas condition (2) is satisfied because $|K|=|G / U|=2$. Thus the hypothesis of Theorem 8.1 is satisfied.

Suppose that we are in case (2). To define the $\mathcal{A}$-groups from the hypothesis of Theorem 8.1 we have to do preliminary work. Set $M$ to be the minimal $\mathcal{A}$-subgroup of $G$ that contains $G_{2}$, and $N$ to be the maximal $\mathcal{A}$-subgroup of $G_{q}$. We claim that

$$
G_{2} \neq M, \quad M_{q} \leq N, \quad N \neq G_{q} .
$$

Indeed, if $G_{2}=M$, then the radical of the highest basic set in $G_{2}$ has trivial $q$-part. However, this is impossible because $\mathcal{A}$ is the $U / L$-wreath product. Similarly, if $N=G_{q}$, then the radical of the highest basic set in $G_{q}$ has trivial 2-part. However, this is impossible because $\mathcal{A}$ is the $V / K$-wreath product. 
To prove the rest we observe that by Theorem 5.2 the S-ring $\mathcal{A}$ is not a $U / K$ wreath product. Then by statement (1) of Corollary 3.3 with $p=2$ there exists an $\mathcal{A}$-group $H$ such that $G_{2} \leq H$ and $H_{q}$ is an $\mathcal{A}$-group. So $M_{q} \leq H_{q}$ and $N \geq H_{q}$ by the choice of $M$ and $N$ respectively. This proves the claim.

Let us verify that the hypothesis of Theorem 8.1 is satisfied for $\mathcal{A}$-groups $K, \widetilde{L}, M, N, U, \widetilde{V}$ where

$$
\widetilde{L}=M \cap N \quad \text { and } \quad \widetilde{V}=M N .
$$

Then obviously $\widetilde{L} \leq N$ and $M \leq \widetilde{V}$. Moreover, from (16) it also follows that $H_{1} \leq H_{2}$ where $H_{1}=K \widetilde{L}$ and $H_{2}=\widetilde{V} \cap U$. Since also

$$
H_{2}=K \times N \text { and } G / H_{1}=M / H_{1} \times U / H_{1} \text {, }
$$

the relations (23) and (24) hold. A part of the $\mathcal{A}$-group lattice is given at Fig. 2,

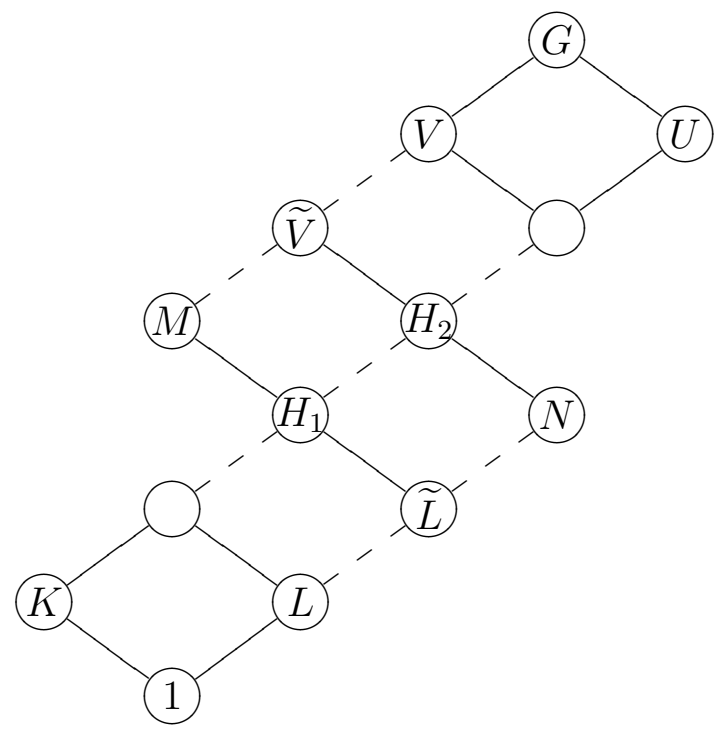

Figure 2:

To verify the rest of the hypothesis of Theorem 8.1 we observe that condition (2) is satisfied because $|K|=|G / U|=2$. We claim that

$$
\mathcal{A}=\mathcal{A}_{U} \imath_{U / \widetilde{L}} \mathcal{A}_{G / \widetilde{L}}
$$


Suppose on the contrary that this is not true. Then there exists a basic set $X$ outside $U$ such that $\operatorname{rad}(X)_{q}<\widetilde{L}$. Then obviously $M^{\prime}:=G_{2} \operatorname{rad}(X)$ is a proper subgroup of $M$ that contains $G_{2}$, which is an $\mathcal{A}$-group by Corollary 3.2 . However, this contradicts the minimality of $M$. Next, let us verify that

$$
\mathcal{A}_{U}=\mathcal{A}_{H_{2}} 2_{H_{2} / K} \mathcal{A}_{U / K} \quad \text { and } \quad \mathcal{A}_{G / \widetilde{L}}=\mathcal{A}_{\widetilde{V} / \widetilde{L}} \tau_{\widetilde{V} / H_{1}} \mathcal{A}_{G / H_{1}} .
$$

To prove the first equality suppose on the contrary that the S-ring $\mathcal{A}_{U}$ is not the $H_{2} / K$-wreath product. Then by statement (1) of Corollary 3.3 with $p=2$ there exists an $\mathcal{A}_{U}$-group $H \not \subset H_{2}$ such that $H_{q}$ is an $\mathcal{A}$-group. However, this is impossible by the maximality of $N$. The second equality can be proved in a similar way. Thus by Remark 8.2 we only have to prove that

$$
\operatorname{rad}\left(\mathcal{A}_{H_{2} / K}\right)=1, \quad \operatorname{rad}\left(\mathcal{A}_{U / H_{1}}\right)=1, \quad \operatorname{rad}\left(\mathcal{A}_{H_{2} / H_{1}}\right)=1
$$

We observe that the third equality follows from the first one and Corollary 7.4 for $G=H_{2} / K$. To prove the first equality in (20) suppose on the contrary that $\operatorname{rad}\left(\mathcal{A}_{H_{2} / K}\right)>1$. To get a contradiction we use the idea from the proof of case (1) in Theorem 5.1. First, we observe that by Corollary 7.2 we have

$$
\mathcal{A}_{H_{2}}=\mathcal{A}_{N} \otimes \mathcal{A}_{K}
$$

Set $U^{\prime}=N^{\prime} K$ where $N^{\prime}$ is the maximal $\mathcal{A}$-group such that $L \leq N^{\prime} \leq N$ and $\operatorname{rad}\left(\mathcal{A}_{N^{\prime}}\right)=1$. Then $N^{\prime}<N$ by the above supposition and the fact that $\mathcal{A}_{N} \cong \mathcal{A}_{H_{2} / K}$. Next, let $X$ be a basic set outside $U^{\prime}$. Then $L \leq \operatorname{rad}(X)$ for $X \subset G \backslash U$ because $\mathcal{A}$ is the $U / L$-wreath product and for $X \subset H_{2} \backslash U^{\prime}$ by the definition of $U^{\prime}$. The same is also true for $X \subset U \backslash H_{2}$. Indeed, otherwise set $Q=\langle X\rangle / \operatorname{rad}(X)$ and $S$ to be the image of the section $H_{2} / K$ in $Q$. Then $\operatorname{rad}\left(\mathcal{A}_{S}\right)=1$ by Theorem 7.3 applied to the S-ring $\mathcal{A}_{Q}$ and the section $S$. On the other hand, $\operatorname{rad}\left(\mathcal{A}_{S}\right)>1$ because $\operatorname{rad}\left(\mathcal{A}_{H_{2} / K}\right)>1$ and $\operatorname{rad}(X) \leq K$. Contradiction. Thus the S-ring $\mathcal{A}$ is the $U^{\prime} / L$-wreath product. Besides, $\operatorname{rad}\left(\mathcal{A}_{U^{\prime}}\right)=1$ because $\operatorname{rad}\left(\mathcal{A}_{N^{\prime}}\right)=1$ and $A_{U^{\prime}}=\mathcal{A}_{N^{\prime}} \otimes \mathcal{A}_{K}$. By statement (2) of Theorem 7.3 for $G=U^{\prime}$ this implies that $\operatorname{rad}\left(\mathcal{A}_{U^{\prime} / L}\right)=1$. However, this contradicts statement (2) of Lemma 4.2. The second equality in (20) is proved similarly following the proof of case (2) in Theorem 5.1.

\section{Auxiliary statements on S-rings}

Given an S-ring $\mathcal{A}$ over a group $G$ we define an $\mathcal{A}$-complement of an $\mathcal{A}$ group $H$ to be an $\mathcal{A}$-group $H^{\prime}$ such that $G=H \times H^{\prime}$. When the group $G$ is 
cyclic, the group $H^{\prime}$ is obviously uniquely determined.

Theorem 7.1 Let $\mathcal{A}$ be an $S$-ring over a cyclic group $G$. Suppose that an $\mathcal{A}$ group $H$ has an $\mathcal{A}$-complement and $\mathcal{A}_{S}=\mathbb{Z} S$ where $S$ is an $\mathcal{A}$-section projectively equivalent to $G / H$. Then given an $\mathcal{A}$-section $T$ projectively equivalent to $H$ the $S$-rings $\mathcal{A}$ and $\mathcal{A}_{S} \otimes \mathcal{A}_{T}$ are Cayley isomorphic.

Proof. Denote by $H^{\prime}$ the $\mathcal{A}$-complement of $H$. Then obviously $H^{\prime} / 1$ and $G / H$ are respectively the smallest and greatest $\mathcal{A}$-sections in the class of projectively equivalent $\mathcal{A}$-sections that contains $G / H$. This implies that the section $S$ is projectively equivalent to (in fact, a multiple of) $H^{\prime} / 1$. By Theorem A3.2 the S-rings $\mathcal{A}_{S}$ and $\mathcal{A}_{H^{\prime}}$ as well as $\mathcal{A}_{T}$ and $\mathcal{A}_{H}$ are Cayley isomorphic. Thus without loss of generality we can assume that $S=H^{\prime} / 1$ and $T=H / 1$. Then $\mathcal{A}_{H^{\prime}}=\mathbb{Z} H^{\prime}$, and hence

$$
\operatorname{rk}(\mathcal{A})=\left|H^{\prime}\right| \operatorname{rk}\left(\mathcal{A}_{H}\right)=\operatorname{rk}\left(\mathcal{A}_{H^{\prime}}\right) \operatorname{rk}\left(\mathcal{A}_{H}\right) .
$$

Since also $\mathcal{A} \geq \mathcal{A}_{H} \otimes \mathcal{A}_{H^{\prime}}$ by Lemma A2.1, we have $\mathcal{A}=\mathcal{A}_{H} \otimes \mathcal{A}_{H^{\prime}}$.

Corollary 7.2 Theorem 7.1 remains true with the condition $\mathcal{A}_{S}=\mathbb{Z} S$ replaced by $|S|=2$.

Some parts of the following statement appeared in a number of papers. Here we formulate it in a more or less general form because it is used throughout the paper several times.

Theorem 7.3 Let $\mathcal{A}$ be a cyclotomic $S$-ring with trivial radical over a cyclic group $G$. Suppose that $S$ is an $\mathcal{A}$-section such that $S_{p} \neq 1$ for any odd prime divisor $p$ of $|G|$. Then

(1) $\left|\operatorname{rad}\left(\mathcal{A}_{S}\right)\right| \leq 2$,

(2) $\left|\operatorname{rad}\left(\mathcal{A}_{S}\right)\right|=1$ unless $\left|S_{2}\right|=4$.

Proof. By [6, Lemma 3.5] given a set $X \in \mathcal{S}(\mathcal{A})$ with $\operatorname{rad}(X)=1$ and a prime $p$ such that $p^{2}$ divides $m=|\langle X\rangle|$, we have $\operatorname{rad}\left(X^{p}\right)=1$ unless $p=2$ and $m=8 m^{\prime}$ with $m^{\prime}$ odd. This shows that $\operatorname{rad}\left(\mathcal{A}_{U}\right)=1$ where $U$ is the subgroup of $G$ of index $p$, unless $p=2$ and $|G|=8 m^{\prime}$ with $m^{\prime}$ odd. Since $\mathcal{A}_{G / L} \cong \mathcal{A}_{U}$ where $L$ is the subgroup of $G$ of order $p$, we have $\mathcal{A}_{G / L}=1$ under 
the same conditions. Thus recursively applying these results we reduces the lemma to the case

$$
\left|S_{2}\right| \leq 4, \quad\left|G_{2}\right| \leq 8, \quad S_{2^{\prime}}=G_{2^{\prime}}
$$

However, from [6, Proposition 3.1] with $m=|G|$ and $l=\left|G_{2^{\prime}}\right|$ it follows that $\operatorname{rad}\left(\mathcal{A}_{G_{2^{\prime}}}\right)=1$. On the other hand, $\operatorname{rad}\left(\mathcal{A}_{S}\right) \leq \operatorname{rad}\left(\mathcal{A}_{S_{2}}\right) \operatorname{rad}\left(\mathcal{A}_{S_{2^{\prime}}}\right)$ because $\mathcal{A}_{S} \geq \mathcal{A}_{S_{2}} \otimes \mathcal{A}_{S_{2^{\prime}}}$ (see Lemma A2.2). Thus $\operatorname{rad}\left(\mathcal{A}_{S}\right) \leq \operatorname{rad}\left(\mathcal{A}_{S_{2}}\right)$, and we are done.

From Theorems A4.1, A4.2 and 7.3 we immediately obtain the following useful result.

Corollary 7.4 Let $\mathcal{A}$ be an $S$-ring with trivial radical over a cyclic p-group, $p$ odd. Then $\operatorname{rad}\left(\mathcal{A}_{S}\right)=1$ for any $\mathcal{A}$-section $S$.

The following statement gives a necessary and sufficient condition for the schurity of an $U / L$-wreath product when the section $U / L$ is one of two sections forming an isolated pair of sections in the corresponding S-ring (see Definition A6.1).

Theorem 7.5 Let $\mathcal{A}=\mathcal{A}_{U} l_{U / L} \mathcal{A}_{G / L}$ be an S-ring over a cyclic group $G$. Suppose that either $\mathcal{A}_{U} \cong \mathcal{A}_{L} \otimes \mathcal{A}_{U / L}$ or $\mathcal{A}_{G / L} \cong A_{U / L} \otimes \mathcal{A}_{G / U}$. Then the $S$-ring $\mathcal{A}$ is schurian if and only if so are the $S$-rings $\mathcal{A}_{U}$ and $\mathcal{A}_{G / L}$.

Proof. The necessity is obvious because given an $\mathcal{A}$-section $S$ the S-ring $\mathcal{A}_{S}$ is schurian whenever so is $\mathcal{A}$. Let us prove the sufficiency under the assumption $\mathcal{A}_{U} \cong \mathcal{A}_{L} \otimes \mathcal{A}_{U / L}$ (the rest can be proved analogously). Denote by $f: U \rightarrow L \times(U / L)$ the corresponding Cayley isomorphism. Then $L^{f}=L$ and $H^{f}=U / L$ for a uniquely determined $\mathcal{A}$-group $H$. It follows that $\mathcal{A}_{U}=$ $\mathcal{A}_{L} \otimes \mathcal{A}_{H}$. Set

$$
\Delta_{0}=\operatorname{Aut}\left(\mathcal{A}_{G / L}\right) \quad \text { and } \quad \Delta_{1}=\operatorname{Aut}\left(\mathcal{A}_{L}\right) \otimes \Delta_{H} .
$$

where $\Delta_{H}$ is the full $f^{U / L}$-preimage of the group $\left(\Delta_{0}\right)^{U / L}$ in the group $\operatorname{Aut}\left(\mathcal{A}_{H}\right)$. Clearly,

$$
(G / L)_{\text {right }} \leq \Delta_{0}, \quad U_{\text {right }} \leq \Delta_{1}, \quad\left(\Delta_{0}\right)^{U / L}=\left(\Delta_{1}\right)^{U / L} .
$$

Moreover, by the schurity of the S-ring $\mathcal{A}_{G / L}$ the latter group is 2-equivalent to the group $\operatorname{Aut}\left(\mathcal{A}_{U / L}\right)$. It follows that the groups $\Delta_{H}$ and $\operatorname{Aut}\left(\mathcal{A}_{H}\right)$, and are 2-equivalent. So by the schurity of the S-ring $\mathcal{A}_{U}$ the groups $\Delta_{1}$ and $\operatorname{Aut}\left(\mathcal{A}_{U}\right)=\operatorname{Aut}\left(\mathcal{A}_{L}\right) \otimes \operatorname{Aut}\left(\mathcal{A}_{H}\right)$ are also 2-equivalent. Thus by Theorem A1.2 the S-ring $\mathcal{A}$ is schurian and we are done. 


\section{A special generalized wreath product}

In this section under special conditions we prove a necessary and sufficient condition for a $U / L$-wreath product to be schurian when the restriction of it to $U / L$ is also a generalized wreath product. We start with the description of elements of the canonical generalized wreath product introduced in Definition A5.32.

Let $G$ be an abelian group and $L \leq U \leq G$. Suppose we are given groups $\Delta_{0} \leq \operatorname{Sym}(G / L)$ and $\Delta_{1} \leq \operatorname{Sym}(U)$ such that $U / L$ is both $\Delta_{0^{-}}$and $\Delta_{1}$-section and

$$
(G / L)_{\text {right }} \leq \Delta_{0}, \quad U_{\text {right }} \leq \Delta_{1}, \quad\left(\Delta_{0}\right)^{U / L}=\left(\Delta_{1}\right)^{U / L}
$$

Then an element of the the canonical generalized wreath product

$$
\Gamma=\Delta_{1} \imath_{U / L} \Delta_{0}
$$

can explicitly be described as follows. Let us fix bijections $h_{X} \in\left(G_{\text {right }}\right)^{U, X}$ where $X \in G / U$. Suppose we are given a permutation $f_{0} \in \Delta_{0}$ and a family $\left\{f_{X} \in \Delta_{1}: X \in G / U\right\}$ of permutations such that

$$
\left(f_{X}\right)^{U / L}=\left(h_{X}\right)^{U / L} f_{0}^{X / L}\left(\left(h_{X^{\prime}}\right)^{U / L}\right)^{-1}
$$

for all $X \in G / U$ where $X^{\prime}$ is the $U$-coset for which $X^{\prime} / L=(X / L)^{f_{0}}$. Then obviously there exists a uniquely determined permutation $f \in \operatorname{Sym}(G)$ for which

$$
f^{G / L}=f_{0} \quad \text { and } \quad f^{X}=\left(h_{X}\right)^{-1} f_{X} h_{X^{\prime}}
$$

for all $X \in G / U$. We stress that this permutation depends on the choice of the permutations $h_{X}$. Denote it by $\left\{f_{X}\right\} \imath_{U / L} f_{0}$. Then the definition of the generalized wreath product of permutation groups implies immediately that

$$
\Gamma=\left\{\left\{f_{X}\right\} \imath_{U / L} f_{0}: f_{0} \in \Delta_{0}, f_{X} \in \Delta_{1} \text { for all } X \in G / U\right\} \text {. }
$$

Let us turn to the main theorem of this section. Let $\mathcal{A}$ be a quasidense $\mathrm{S}-$ ring over a cyclic group $G$. Suppose we are given $\mathcal{A}$-groups $K, L, M, N, U, V$ such that $L \leq N, M \leq V$,

$$
H_{1}:=K L \leq U \cap V:=H_{2}
$$




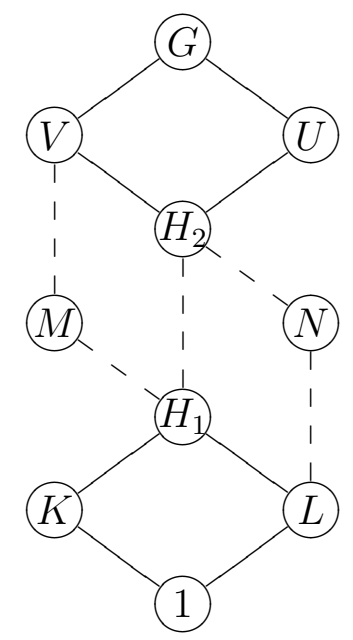

Figure 3:

and also

$$
H_{2}=K \times N \text { and } G / H_{1}=M / H_{1} \times U / H_{1} .
$$

The corresponding part of the $\mathcal{A}$-group lattice is represented in Fig. 3 ,

Theorem 8.1 In the above notation suppose that the $S$-rings $\mathcal{A}, \mathcal{A}_{U}$ and $\mathcal{A}_{G / L}$ are respectively the $U / L-, H_{2} / K$ - and $V / H_{1}$-wreath products such that

(1) the $S$-rings $\mathcal{A}_{H_{2} / K}, \mathcal{A}_{U / H_{1}}$ and $\mathcal{A}_{V / H_{1}}, \mathcal{A}_{H_{2} / L}$ are of trivial radicals,

(2) $\mathcal{A}_{K}=\mathbb{Z} K$ and $\mathcal{A}_{G / U}=\mathbb{Z} G / U$.

Then the $S$-ring $\mathcal{A}$ is schurian if and only if so are the $S$-rings $\mathcal{A}_{U}$ and $\mathcal{A}_{G / L}$.

Remark 8.2 Equalities (24) together with condition (2) imply by Theorem 7.1 that $\mathcal{A}_{V / H_{1}} \cong \mathcal{A}_{G / U} \otimes \mathcal{A}_{H_{2} / H_{1}}$ and $\mathcal{A}_{H_{2} / L} \cong \mathcal{A}_{K} \otimes \mathcal{A}_{H_{2} / H_{1}}$. Thus in our case the second part of condition (1) is equivalent to the equality $\operatorname{rad}\left(\mathcal{A}_{H_{2} / H_{1}}\right)=1$.

Proof. The necessity is obvious. To prove the sufficiency suppose that the S-rings $\mathcal{A}_{U}$ and $\mathcal{A}_{G / L}$ are schurian. Set

$$
\Gamma_{1}=\operatorname{Hol}_{\mathcal{A}}\left(U / H_{1}\right) \quad \text { and } \quad \Gamma_{2}=\operatorname{Hol}_{\mathcal{A}}\left(H_{2} / L\right) .
$$

\footnotetext{
${ }^{2}$ The group that was denoted there by $\Delta_{U}$ is denoted here by $\Delta_{1}$.
} 
Then obviously $\left(\Gamma_{1}\right)^{H_{2} / H_{1}}=\left(\Gamma_{2}\right)^{H_{2} / H_{1}}$. So one can define the generalized wreath product $\Delta=\Gamma_{2} \imath_{H_{2} / H_{1}} \Gamma_{1}$. Thus by Theorem A1.2 to complete the proof it suffices to find groups $\Delta_{1} \in \mathcal{M}\left(\mathcal{A}_{U}\right)$ and $\Delta_{0} \in \mathcal{M}\left(\mathcal{A}_{G / L}\right)$ such that

$$
\left(\Delta_{1}\right)^{U / L}=\Delta=\left(\Delta_{0}\right)^{U / L} .
$$

To do this we observe that by Theorem 3.5 and due to condition (1) there exist groups $\Gamma_{3} \in \mathcal{M}\left(\mathcal{A}_{U / K}\right)$ and $\Gamma_{6} \in \mathcal{M}\left(\mathcal{A}_{V / L}\right)$ such that

$$
\begin{aligned}
&\left(\Gamma_{3}\right)^{H_{2} / K}=\operatorname{Hol}_{\mathcal{A}}\left(H_{2} / K\right),\left(\Gamma_{3}\right)^{U / H_{1}}=\operatorname{Hol}_{\mathcal{A}}\left(U / H_{1}\right), \\
&\left(\Gamma_{6}\right)^{V / H_{1}}=\operatorname{Hol}_{\mathcal{A}}\left(V / H_{1}\right), \quad\left(\Gamma_{6}\right)^{H_{2} / L}=\operatorname{Hol}_{\mathcal{A}}\left(H_{2} / L\right) .
\end{aligned}
$$

Set

$$
\Gamma_{4}=\operatorname{Hol}_{\mathcal{A}}\left(H_{2}\right), \quad \Gamma_{5}=\operatorname{Hol}_{\mathcal{A}}\left(G / H_{1}\right) .
$$

Then clearly $\left(\Gamma_{4}\right)^{H_{2} / K}=\left(\Gamma_{3}\right)^{H_{2} / K}$ and $\left(\Gamma_{6}\right)^{V / H_{1}}=\left(\Gamma_{5}\right)^{V / H_{1}}$. Therefore one can define generalized wreath products

$$
\Delta_{1}=\Gamma_{4} \imath_{H_{2} / K} \Gamma_{3} \text { and } \Delta_{0}=\Gamma_{6} \imath_{V / H_{1}} \Gamma_{5} \text {. }
$$

First, let us prove that $\Delta_{1} \in \mathcal{M}\left(\mathcal{A}_{U}\right)$ and $\Delta_{0} \in \mathcal{M}\left(\mathcal{A}_{G / L}\right)$. Indeed, since $U_{\text {right }} \leq \Delta_{1}$ and $(G / L)_{\text {right }} \leq \Delta_{0}$, it suffices to verify that

$$
\Delta_{1} \underset{2}{\approx} \operatorname{Aut}\left(\mathcal{A}_{U}\right) \text { and } \Delta_{0} \underset{2}{\approx} \operatorname{Aut}\left(\mathcal{A}_{G / L}\right)
$$

In its turn, to prove these relations it suffices to verify by Corollary A5.7 applied to the S-ring $\mathcal{A}_{U}$ and the groups $\Gamma_{4}, \Gamma_{3}$, and the S-ring $\mathcal{A}_{G / L}$ and the groups $\Gamma_{6}, \Gamma_{5}$, that

$$
\Gamma_{4} \underset{2}{\approx} \operatorname{Aut}\left(\mathcal{A}_{H_{2}}\right), \Gamma_{3} \underset{2}{\approx} \operatorname{Aut}\left(\mathcal{A}_{U / K}\right), \Gamma_{6} \underset{2}{\approx} \operatorname{Aut}\left(\mathcal{A}_{V / L}\right), \Gamma_{5} \underset{2}{\approx} \operatorname{Aut}\left(\mathcal{A}_{G / H_{1}}\right) .
$$

However, the statements on $\Gamma_{3}$ and $\Gamma_{6}$ hold by the definition of these groups. Next, the hypothesis $\mathcal{A}_{K}=\mathbb{Z} K$ implies by Theorem 7.1 that $\mathcal{A}_{H_{2}}$ is the tensor product of the cyclotomic rings $\mathcal{A}_{K}$ and $\mathcal{A}_{N} \cong \mathcal{A}_{H_{2} / K}$ (the latter Sring is cyclotomic by Theorem 3.1). Therefore the S-ring $\mathcal{A}_{H_{2}}$ is cyclotomic, and hence the groups $\operatorname{Aut}\left(\mathcal{A}_{H_{2}}\right)$ and $\Gamma_{4}$ are 2-equivalent. Similarly, one can prove that the group $\Gamma_{5}$ is 2 -equivalent to the group $\operatorname{Aut}\left(\mathcal{A}_{G / H_{1}}\right)$.

To prove (25) we note that due to (26), (27) and (28) we have

$$
\left(\Gamma_{3}\right)^{U / H_{1}}=\Gamma_{1}=\left(\Gamma_{5}\right)^{U / H_{1}} \quad \text { and } \quad\left(\Gamma_{4}\right)^{H_{2} / L}=\Gamma_{2}=\left(\Gamma_{6}\right)^{H_{2} / L} .
$$


Therefore both $\left(\Delta_{1}\right)^{U / L}$ and $\left(\Delta_{0}\right)^{U / L}$ are contained in the group $\Delta$. To prove the converse inclusion we observe that due to (24) there is an isomorphism

$$
G / V \rightarrow U / H_{2}, \quad X \mapsto X \cap U=: Y .
$$

In what follows the factor sets $X$ and $Y$ modulo $L$ are denoted by $\bar{X}$ and $\bar{Y}$ respectively. For each $X \in G / V$ we fix a bijection $h_{X} \in\left(G_{\text {right }}\right)^{V, X}$ that takes $H_{2}$ to $Y$, and set

$$
h_{Y}=\left(h_{X}\right)^{Y}, \quad h_{\bar{X}}=\left(h_{X}\right)^{\bar{X}}, \quad h_{\bar{Y}}=\left(h_{X}\right)^{\bar{Y}} .
$$

Then due to equality (22) any element $\bar{f} \in \Delta$ can be written in the form

$$
\bar{f}=\left\{f_{\bar{Y}}\right\} 2_{H_{2} / H_{1}} \bar{f}_{0}
$$

for some permutation $\bar{f}_{0} \in \Gamma_{1}$ and a family of permutations $f_{\bar{Y}} \in \Gamma_{2}$ where $Y \in U / H_{2}$, such that

$$
\left(f_{\bar{Y}}\right)^{H_{2} / H_{1}}=\left(h_{\bar{Y}}\right)^{H_{2} / H_{1}}\left(\bar{f}_{0}\right)^{Y / H_{1}}\left(\left(h_{\overline{Y^{\prime}}}\right)^{H_{2} / H_{1}}\right)^{-1}
$$

for all $Y \in U / H_{2}$ where $Y^{\prime}$ is the $H_{2}$-coset in $U$ for which $Y^{\prime} / H_{1}=\left(Y / H_{1}\right)^{\bar{f}_{0}}$. In what follows we find some elements of the groups $\Delta_{1}$ and $\Delta_{0}$ the restrictions of which to $U / L$ coincide with $\bar{f}$.

To find the required permutation in $\Delta_{0}$ we observe that $\mathcal{A}_{M / H_{1}}=\mathbb{Z} M / H_{1}$ because $\mathcal{A}_{M / H_{1}} \cong \mathcal{A}_{G / U}$, and the latter is a group ring by condition (2). So by Theorem 7.1 we have

$$
\Gamma_{5}=\left(M / H_{1}\right)_{\text {right }} \otimes \Gamma_{1}
$$

Therefore this group contains the permutation $f_{0}=\mathrm{id}_{M / H_{1}} \otimes \bar{f}_{0}$. Clearly,

$$
\left(f_{0}\right)^{U / H_{1}}=\bar{f}_{0} \text {. }
$$

Next, let $X \in G / V$. Then by (27) there exists a permutation $f_{\bar{X}} \in \Gamma_{6}$ that leaves the set $H_{2} / L$ fixed and such that

$$
\left(f_{\bar{X}}\right)^{H_{2} / L}=f_{\bar{Y}} .
$$

Below we show that

$$
\left(f_{\bar{X}}\right)^{V / H_{1}}=\left(h_{\bar{X}}\right)^{V / H_{1}}\left(f_{0}\right)^{X / H_{1}}\left(\left(h_{\overline{X^{\prime}}}\right)^{V / H_{1}}\right)^{-1}
$$


where $X^{\prime}$ is the $V$-coset in $G$ for which $X^{\prime} / H_{1}=\left(X / H_{1}\right)^{f_{0}}$. Then one can define a permutation $f=\left\{f_{\bar{X}}\right\}_{\imath_{V / H_{1}}} f_{0}$ belonging to the group $\Delta_{0}$. This is we wanted to find because $f^{U / L}=\bar{f}$ by (31), (34) and (35).

To prove (36) we observe that $\left(\Gamma_{6}\right)^{V / H_{1}}=\left(V / H_{2}\right)_{\text {right }} \otimes \operatorname{Hol}_{\mathcal{A}}(V / M)$ because $\mathcal{A}_{V / H_{2}}=\mathbb{Z} V / H_{2}$ (see above). So

$$
\left(f_{\bar{X}}\right)^{V / H_{1}}=\left(f_{\bar{X}}\right)^{V / H_{2}} \otimes\left(f_{\bar{X}}\right)^{V / M}=\left(f_{\bar{X}}\right)^{V / H_{2}} \otimes\left(f_{\bar{Y}}\right)^{V / M} .
$$

On the other hand, the permutation $f_{\bar{X}}$ leaves the set $H_{2} / L$ fixed. So $\left(f_{\bar{X}}\right)^{V / H_{2}}$ lives the set $H_{2}$ fixed. Since also $\left(f_{\bar{X}}\right)^{V / H_{2}} \in\left(V / H_{2}\right)_{\text {right }}$, this implies that $\left(f_{\bar{X}}\right)^{V / H_{2}}=\mathrm{id}_{V / H_{2}}$. Thus (36) holds by (32) and the choice of the bijections $h_{X}$.

To find a permutation $f \in \Delta_{1}$ such that $f^{U / L}$ coincides with the permutation $\bar{f}$ defined in (30), we observe that due to (26) there exists a permutation $f_{0} \in \Gamma_{3}$ such that equality (34) holds. Next, for each $Y \in U / H_{2}$ we define a permutation of $\mathrm{H}_{2} / \mathrm{K}$ defined by

$$
g_{Y}=\left(h_{Y}\right)^{H_{2} / K}\left(f_{0}\right)^{Y / K}\left(\left(h_{Y^{\prime}}\right)^{H_{2} / K}\right)^{-1}
$$

where $Y^{\prime}$ is the $H_{2}$-coset in $U$ for which $Y^{\prime} / K=(Y / K)^{f_{0}}$. However, the bijection $h_{X}$ by its choice leaves the set $U$ fixed. So

$$
\left(h_{Y}\right)^{H_{2} / K}\left(f_{0}\right)^{Y / K}\left(\left(h_{Y^{\prime}}\right)^{H_{2} / K}\right)^{-1}=\left(\left(h_{X}\right)^{U / K} f_{0}\left(\left(h_{X^{\prime}}\right)^{U / K}\right)^{-1}\right)^{H_{2} / K}
$$

Thus $g_{Y}$ belongs to the group $\left(\Gamma_{3}\right)^{H_{2} / K}=\left(\Gamma_{4}\right)^{H_{2} / K}$. Therefore, due to condition (2) of the theorem the permutation

$$
f_{Y}:=g_{Y} \otimes\left(f_{\bar{Y}}\right)^{H_{2} / N}
$$

belongs to the group $\Gamma_{4}$. Moreover, equalities (34), (37) and (32) imply that

$$
\left.\left(f_{Y}\right)^{H_{2} / L}=\left(h_{Y}\right)^{H_{2} / L}\left(f_{0}\right)^{Y / L}\left(h_{Y^{\prime}}\right)^{H_{2} / L}\right)^{-1} .
$$

Thus one can define a permutation $f=\left\{f_{Y}\right\} \imath_{H_{2} / L} f_{0}$ belonging to the group $\Delta_{1}$. By the choice of $f_{0}$ and $f_{Y}$ we have $f^{U / L}=\bar{f}$, which completes the proof.m 


\section{References}

[1] S. Evdokimov, I. Ponomarenko, On a family of Schur rings over a finite cyclic group, Algebra and Analysis, 13 (2001), 3, 139-154.

[2] S. Evdokimov, I. Ponomarenko, Characterization of cyclotomic schemes and normal Schur rings over a cyclic group, Algebra and Analysis, 14 (2002), 2, 11-55.

[3] S. Evdokimov, I. Ponomarenko, Schurity of S-rings over a cyclic group and generalized wreath product of permutation groups, Algebra and Analysis, 24 (2012), 3, 84-127.

[4] M. Kh. Klin, R. Pöschel, The König problem, the isomorphism problem for cyclic graphs and the method of Schur rings, Algebraic Methods in Graph Theory. Vol. 1, 2 (Szeged, 1978), Colloq. Math. Soc. János Bolyai, vol. 25, North-Holland, Amsterdam-New York, 1981, pp. 405-434.

[5] I. Kovács, A construction of the automorphism groups of indecomposable S-rings over $\mathbb{Z}_{2^{n}}$, Beitr. Algebra Geom., 52 (2011), no. 1, 83-103.

[6] K. H. Leung, S. H. Man, On Schur Rings over Cyclic Groups, II, J. Algebra, 183 (1996), 273-285.

[7] R. Pöschel, Untersuchungen von S-Ringen insbesondere im Gruppenring von p-Gruppen, Math. Nachr., 60 (1974), 1-27.

[8] I. Schur, Zur Theorie der einfach transitiven Permutationgruppen, S.-B. Preus Akad. Wiss. Phys.-Math. Kl., (1933), 598-623.

[9] H. Wielandt, Finite permutation groups, Academic press, New York London, 1964. 